$\Rightarrow$ CARDIOMYOPATHY

\title{
Takotsubo has long-lasting functional consequences
}

Takotsubo cardiomyopathy, an acute heart failure syndrome triggered by an emotionally stressful episode, was believed to be a transient and self-limiting condition, because patients have spontaneous recovery of left ventricular ejection fraction (LVEF).

Dana Dawson and colleagues now show that Takotsubo cardiomyopathy is associated with long-lasting clinical and functional changes. "This finding represents a paradigm shift in the perspective of how we view patients with this condition," says Dawson. "Patients should be counselled on the long-term symptomatic consequences of Takotsubo cardiomyopathy."

Most of the patients enrolled in the study (88\%) had persisting heart failure symptoms at a median of 20 months after the occurrence of Takotsubo cardiomyopathy, with a median score of 13 in the Minnesota Living with Heart Failure Questionnaire, and had reduced cardiopulmonary exercise capacity compared with control participants matched for age, sex, and comorbidities. Moreover, despite having normal LVEF, these patients had structural and metabolic alterations in the myocardium, as shown by impaired cardiac deformation indices (indicative of cardiac remodelling), higher native $\mathrm{T} 1$ mapping values (indicating development of microscopic fine scarring), and an impaired cardiac energetic status compared with control individuals.

"Taken together, these findings demonstrate a substantial and persistent adverse alteration in the long-term cardiac morbidity associated with Takotsubo cardiomyopathy, and define a new clinical phenotype in this population," concludes Dawson.

Irene Fernández-Ruiz

ORIGINAL ARTICLE Scally, C. et al. Persistent long-term structural, functional, and metabolic changes after stressinduced (Takotsubo) cardiomyopathy. Circulation http:// dx.doi.org/10.1161/CIRCULATIONAHA.117.031841 (2017) FURTHER READING Kastaun, S. et al. Psychosocial and

psychoneuroendocrinal aspects of Takotsubo syndrome. Nat. Rev. Cardiol. 13, 688-694 (2016) 